\title{
Correction to: The delamination of a growing elastic sheet with adhesion
}

\author{
Gaetano Napoli $\cdot$ Stefano Turzi
}

Accepted: 18 November 2020/Published online: 6 January 2021

(C) Springer Nature B.V. 2021

\section{Correction to: Meccanica (2017) 52:3481-3487 https://doi.org/10.1007/s11012-017-0618-0}

We have found two mistakes in Eqs. (13) and (14) of Ref. [1], which we wish to correct. We note that these errors did not produce significant effects on the subsequent numerical analysis, because the corrective term only introduces a negligible contribution to the solution in the region of interest of the parameters.

The error in Eq. (13) is simply a misprint and does not affect the rest of the paper. The correct version of Eq. (13) is

$$
\left(w_{f}-w_{a}-\frac{\partial w_{f}}{\partial \theta^{\prime}} \theta^{\prime}\right) \delta \bar{S}=0 .
$$

Since this equation is requested to vanish for any $\delta \bar{S}$, with the use of Eqs. (5) of Ref. [1], we obtain

The original article can be found online at https:// doi.org/10.1007/s11012-017-0618-0.

\section{G. Napoli}

Dipartimento di Matematica e Fisica "E. De Giorgi",

Università del Salento, Lecce, Italy

e-mail: gaetano.napoli@unisalento.it

\section{S. Turzi $(\square)$}

Dipartimento di Matematica, Politecnico di Milano,

Milan, Italy

e-mail: stefano.turzi@polimi.it

$$
\begin{aligned}
& -k\left[\theta^{\prime}(\bar{S})\right]^{2}+b\left[\left(\lambda^{-}-1\right)^{2}-\left(\lambda^{+}-1\right)^{2}\right] \\
& +2 \mu\left(\lambda^{+}-\lambda^{-}\right)+2 w \lambda^{+}=0
\end{aligned}
$$

where $\lambda^{-}=1+\mu / b$ and $\lambda^{+}=\bar{\lambda}$. Furthermore, Eq. (12) of Ref. [1] leads to $\mu=b(\bar{\lambda}-1)-w$, which inserted into (2) yields the correct version of Eq. (14)

$$
-k\left[\theta^{\prime}(\bar{S})\right]^{2}+2 \bar{\lambda} w-\frac{w^{2}}{b}=0
$$

This can be further simplified using the elastocapillary length $\ell_{e c}$ so that (3) rewrites as

$$
-k\left[\theta^{\prime}(\bar{S})\right]^{2}+2 \bar{\lambda} w-w \frac{\ell^{2}}{\ell_{e c}^{2}}=0 .
$$

In $\$ 3$ of Ref. [1] we studied the case $\ell^{2} / \ell_{e c}^{2} \simeq 1.37 \times 10^{-5}$, so that the corrective term contribution is negligible. We are grateful to Jun Zhong for his remarks.

\section{References}

1. Napoli G, Turzi S (2017) The delamination of a growing elastic sheet with adhesion. Meccanica 52(14):3481-3487

Publisher's Note Springer Nature remains neutral with regard to jurisdictional claims in published maps and institutional affiliations. 